\title{
Macroscopic two-fluid effects in magnetorheological fluids
}

\author{
Harald Pleiner* \\ Max Planck Institute for Polymer Research, 55021 Mainz, Germany \\ Daniel Svenšek \\ Department of Physics, Faculty of Mathematics and Physics, University of Ljubljana, SI-1000 Ljubljana, Slovenia \\ Tilen Potisk $\odot$ and Helmut R. Brand \\ Department of Physics, University of Bayreuth, 95440 Bayreuth, Germany
}

(Received 17 December 2019; accepted 11 February 2020; published 2 March 2020)

\begin{abstract}
We investigate macroscopic two-fluid effects in magnetorheological fluids generalizing a one-fluid model studied before. In the bulk of the paper we use a model in which the carrier fluid, with density $\rho_{1}$, moves with velocity $\boldsymbol{v}_{1}$, while the magnetic component (density $\rho_{2}$ ) and, therefore, the magnetization and the magneticfield-induced relaxing strain field move with velocity $\boldsymbol{v}_{\mathbf{2}}$. In the framework of macroscopic dynamics we find, in particular, reversible dynamic and dissipative cross-coupling terms between the magnetization and the velocity difference. Experiments to detect some of these cross-coupling terms are suggested. We also compare the results of the two-fluid model presented here with two-fluid models available for electrorheological fluids. In two appendices we discuss the simplifying assumptions made to arrive at the model used in this paper and we also outline how to detect potential deviations from this model.
\end{abstract}

DOI: 10.1103/PhysRevE.101.032601

\section{INTRODUCTION}

Two-fluid behavior is abundant in the field of complex fluids such as polymers, elastomers, and microemulsions. Systems of interest include multiphase flows of bubbly liquids and fluid suspensions of particulates [1], viscoelasticity of concentrated fluid emulsions [2], flow-induced ordering of wormlike micellar solutions [3], and flow of colloidal suspensions [4]. Regarding flows of interest for two-fluid models a number of situations for complex fluids come to mind. Examples include dynamical phenomena in polymer solutions and blends associated with hydrodynamics and rheology [5-8] and polymer migration and phase separation under flow [9-12]. In the context of bioinspired nonequilibrium systems a two-fluid model has been set up to derive the active polar two-fluid macroscopic dynamics for systems with a polar dynamic preferred direction [13].

Superfluids are another class of two-fluid systems. But there is a key difference between two-fluid effects in superfluids and those for systems that are not superfluid. In superfluids one has broken gauge symmetry and the operator associated with the particle number acts as the generator of

\footnotetext{
*pleiner@mpip-mainz.mpg.de
}

Published by the American Physical Society under the terms of the Creative Commons Attribution 4.0 International license. Further distribution of this work must maintain attribution to the author(s) and the published article's title, journal citation, and DOI. Open access publication funded by the Max Planck Society. this broken continuous symmetry. In accord with this picture superfluid systems possess two truly hydrodynamic velocities as variables [14-16]. As a consequence the dynamic equations for both the normal and the superfluid velocity do not have a gap even in the long wavelength (hydrodynamic) limit.

This situation must be distinguished from two-fluid effects in systems that are not superfluid. In this case only the barycentric velocity is a truly hydrodynamic variable and has no gap in the excitation spectrum in the long wavelength limit. In contrast, the relative velocity, although long lived and therefore kept on the list of macroscopic variables, acquires a gap for small wave vectors. When it comes to two-fluid effects in systems that are not superfluid, clearly Ref. [17] contains the most detailed and complete derivation of macroscopic equations and their analysis. However, the effects of a magnetization have not been considered there.

It seems that macroscopic equations describing two-fluid effects with two velocities and two densities for magnetorheological fluids (MRFs) have not been considered before. Recently, a one-fluid description of MRFs in the framework of macroscopic dynamics (or generalized hydrodynamics) has been given [18]. Thus, for the purpose of this paper, the derivation of macroscopic two-fluid effects in MRFs, we have to generalize the macroscopic dynamics for isotropic viscoelastic two-fluid systems by including the magnetization as variable, or introduce a second velocity (and density) to the previous macroscopic dynamics of MRFs. One of the advantages of MRFs in comparison to nonmagnetic twofluid systems is the fact that the magnetization can be tuned continuously and controlled by external fields. From the side of a one-fluid description of MRFs one can expect to be able 
to describe various effects in more detail, or cover specific two-fluid effects, when using the two-fluid description for MRFs.

The paper is organized as follows. In Sec. II we present our macroscopic model including the derivation of the static (Sec. II A) and dynamic (Sec. II B) macroscopic equations. In Sec. III we discuss specific two-fluid aspects and possible experiments to test our model. Analogies to the field of two-fluid effects in electrorheological fluids (ERFs) are given in Sec. IV. In several appendices we deal with subtle aspects or lengthy derivations not suitable for the main textin particular, general thermodynamic considerations of twofluid models (Appendix A), a discussion of general transport derivatives and the simplifying assumptions made to arrive at the dynamic equations used in the bulk of the paper (Appendix B), magnetic vorticity and the analogy to superfluid ${ }^{3} \mathrm{He}$ (Appendix C), the ground-state shift due to an external field (including the zero field limit) in Appendix D, and the formal aspects of the reduction of the two-fluid model to a one-fluid description (Appendix E).

\section{MACROSCOPIC MODEL}

First we will discuss the variables necessary to generalize our MRF model [18] into a two-fluid system [17,19]. The basic notion is to have two intrinsically coupled subsystems, a Newtonian background fluid (index 1) and a (visco)elastic magnetic structure due to an applied magnetic field (index 2). They are described by the mass densities $\rho_{1}$ and $\rho_{2}$ and two momentum densities $\boldsymbol{g}_{1}=\rho_{1} \boldsymbol{v}_{1}$ and $\boldsymbol{g}_{2}=\rho_{2} \boldsymbol{v}_{2}$. We will also use the concentration variables, $\phi \equiv \rho_{1} / \rho$ (or $1-\phi \equiv$ $\left.\rho_{2} / \rho\right)$ with $\rho=\rho_{1}+\rho_{2}$ the total density. The latter is a conserved quantity and is related to the total momentum density $\boldsymbol{g}=\boldsymbol{g}_{1}+\boldsymbol{g}_{2}=\rho \boldsymbol{v}$ through the mean velocity $\boldsymbol{v}=\phi \boldsymbol{v}_{1}+(1-$ $\phi) \boldsymbol{v}_{2}$. As the second velocity one can then use $\boldsymbol{w} \equiv \boldsymbol{v}_{1}-\boldsymbol{v}_{2}$, the relative velocity. When using Cartesian components we will write, e.g., $v_{i}^{(1)}$ and $v_{i}^{(2)}$ for the two velocities. The thermodynamics of this two-fluid system is discussed in detail in Appendix A.

As in the one-fluid case we consider the field-induced magnetization $\boldsymbol{M}$ and the field-induced strain field $U_{i j}$ as additional macroscopic variables. The latter is due to elastic structures (e.g. columns of magnetic particles) that build up in an external field. The elasticity is assumed to be relaxing, since when the columns are deformed the particles tend to redistribute (by permeation). On the macroscopic level this manifests as relaxation of the strain and results in viscoelasticity.

Hydrodynamic equations typically exhibit transport (advection and/or convection) contributions involving the velocity of the system [20]. In the two-fluid case the choice of the velocity is not unique. It is tempting, e.g., to assume that the variables $\rho_{1}$ and $\boldsymbol{g}_{1}$ are transported with velocity $\boldsymbol{v}_{1}$, while for $\rho_{2}, \boldsymbol{g}_{2}$, the magnetization $\boldsymbol{M}$, and the relaxing strain field $U_{i j}$ one takes $\boldsymbol{v}_{2}$. This is not the most general case possible, but we will adopt this choice in order to simplify the model. The transport contributions are reversible (in time) and therefore must not increase the entropy of the system. This produces some restrictions (and compensation terms) to be taken care of. In addition, the transport of the total density $\rho$ and the total momentum density $g$ must be done by the mean velocity $\boldsymbol{v}$. These questions will be dealt with comprehensively in Appendix B, while in the main body of the paper we use the resulting simplified equations, which are then compatible with all thermodynamic requirements.

The system has only one thermal degree of freedom described by the entropy density $\sigma$ and its static behavior is governed by the energy density $\varepsilon$. The magnetic degree of freedom is described by the magnetization $\boldsymbol{M}$ and the magnetic induction field $\boldsymbol{B}$. The latter is treated within magnetostatics, and only shows up in a quadratic contribution to the pressure $p$ and in the Maxwell stress [21]. The (visco)elastic properties are described by the relaxing strain field $U_{i j}$. In order to make the model as simple as possible, we will not discuss thermal effects, disregarding $\sigma$ as a dynamic variable, later on. Similarly, we assume the total mass density $\rho$ to be constant, although this approximation is less powerful in the two-fluid case compared to the one-fluid one.

The system becomes uniaxial only due to the external field. We therefore will often take the material tensors as isotropic, but generally they are of uniaxial symmetry.

We have chosen $\rho$ and $\phi$ as variables (rather than $\rho_{1}$ and $\rho_{2}$ ), since the density drops out later anyhow, and only one variable is left. Similarly, we take $g_{i}$ and $w_{i}$ (rather than $g_{i}^{(1)}$ and $\left.g_{i}^{(2)}\right)$, since the total momentum density has a quite simple dynamics, while all the complexity that results from the special choices of the transport velocities accumulates in the $\dot{w}_{i}$ equation; here, it is easy to make additional approximations, since $\dot{w}_{i}$ is in zeroth order a simple relaxation due to the friction term; to do such additional approximations in $\dot{g}_{i}^{(1,2)}$ is much more delicate.

One might be tempted to model ferrofluids in the same way as magnetorheological fluids, since both systems are composed of magnetic particles suspended in a carrier fluid. The important difference is the size of the magnetic particles, which are in the micrometer range for MRFs and are nanometer sized for ferrofluids. Even a small external magnetic field induces large magnetic attractive forces between the particles in MRFs, which form rigid structures capable of resisting to externally imposed shear strains. These columns are the cause for a large static yield stress in MRFs, which is on the other hand negligible in ferrofluids due to the lack of elasticity, except for very large fields. The noncontinuous field dependence of the viscosity of ferrofluids in this intermediate field range has no counterpart in MRFs.

\section{A. Statics}

The Gibbs relation, a manifestation of the first law of thermodynamics, relates changes of the macroscopic variables to energy changes and reads for our model (see Appendix A)

$$
\begin{aligned}
d \varepsilon= & T d \sigma+\mu d \rho+\Pi d \phi+v_{i} d g_{i}+h_{i}^{w} d w_{i} \\
& +h_{i}^{M} d M_{i}+H_{i} d B_{i}+\Phi_{i j} d U_{i j} .
\end{aligned}
$$

The thermodynamic conjugates are the temperature $T$, the mean velocity $v_{i}$, the conjugate quantity to the relative velocity $h_{i}^{w}$, the internal magnetic induction $h_{i}^{M}$, the (external) magnetic field $H_{i}$, the elastic stress $\Phi_{i j}$, and the osmotic pressure П. Rotational invariance of Eq. (1) requires $\Phi_{i k} U_{j k}=\Phi_{j k} U_{i k}$. 
For the thermodynamic pressure we have

$$
p=-\varepsilon+T \sigma+\mu \rho+\Pi \phi+v_{i} g_{i}+h_{i}^{w} w_{i}+B_{i} H_{i}
$$

where $B_{i}=\mu_{0}\left(H_{i}+M_{i}\right)$ is the magnetic flux density.

The statics of a macroscopic system is best set up by considering its total energy density $\varepsilon$ in terms of all variables. According to the Gibbs relation, the conjugates are then partial derivatives of $\varepsilon$.

We split $\varepsilon$ in two parts, the kinetic energy, $\varepsilon_{1}$, and all other contributions, $\varepsilon_{2}$. For the kinetic energy density we have

$$
\varepsilon_{1}=\frac{1}{2} \rho_{1} \boldsymbol{v}_{1}^{2}+\frac{1}{2} \rho_{2} \boldsymbol{v}_{2}^{2}=\frac{1}{2 \rho} \boldsymbol{g}^{2}+\frac{\rho_{1} \rho_{2}}{2 \rho} \boldsymbol{w}^{2},
$$

which immediately gives the conjugates as

$$
v_{i}=\rho^{-1} g_{i} \quad \text { and } \quad h_{i}^{w}=\rho \phi(1-\phi) w_{i}
$$

in accordance with Appendix A.

Using proper time and space symmetries [20] we get for the phenomenological part of the energy density

$$
\begin{aligned}
\varepsilon_{2}= & -\mu_{0} H_{i} M_{i}+\frac{1}{2} \alpha \boldsymbol{M}^{2}+\frac{1}{4} \beta\left(\boldsymbol{M}^{2}\right)^{2} \\
& +\frac{1}{2} \tilde{c}_{i j k l} U_{i j} U_{k l}-\frac{1}{2} \gamma_{i j k l} U_{i j} M_{k} M_{l} \\
& +\frac{1}{2} \chi_{\phi} \bar{\phi}^{2}+\tilde{\chi}_{u} U_{k k} \bar{\phi}+\chi_{m} \boldsymbol{M}^{2} \bar{\phi}
\end{aligned}
$$

where we have already omitted changes of the total density and the thermal degree of freedom. The quantity $\bar{\phi}$ is the excess concentration beyond the equilibrium value $\rho_{1} / \rho$. Inspecting Eqs. (10)-(12) reveals that the variables $\bar{\phi}, M_{i}$, and $U_{i j}$ are coupled to each other statically.

The $\beta$ term can be viewed as the next order $M^{2}$ correction to $\alpha$. The form given in Eq. (5) is suitable for rather small fields, while in the general case the $\alpha$ and $\beta$ terms have to be replaced by a more complicated function $f_{1}\left(M^{2}\right)$ that can be taken from experimental results. The contribution $\sim \chi_{m}$, coupling $\delta \phi$ and $\boldsymbol{M}^{2}$, is the lowest order $\delta \phi$ correction of $\alpha$. In principle, $\alpha$ could have a more general $\delta \phi$ dependence. The coupling $\sim \tilde{\chi}_{u}$ is an example of couplings between scalar variables (e.g. $\delta \phi$ ) and rotational invariants of tensors, the trace $U_{k k}$, and, e.g., the twist in cholesteric liquid crystals.

In Eq. (5) a coupling between the relative velocity and the magnetic vorticity (or between the magnetization and the vorticity of the relative velocity), $\sim \epsilon_{i j k} w_{i} \nabla_{j} M_{k}$, allowed by symmetry, has to be discarded, since it is incompatible with the assumption of our simplified model that $g_{1}$ and $\boldsymbol{g}_{2}$ are only related to $\boldsymbol{v}_{1}$ and $\boldsymbol{v}_{2}$, respectively. In Appendix $\mathrm{C}$ we dwell on some implications of this coupling and compare with ${ }^{3} \mathrm{He}-\mathrm{A}$, where a similar coupling exists.

The material tensors $\tilde{c}_{i j k l}$ and $\gamma_{i j k l}$ describe elasticity and magnetostriction, respectively. They have the uniaxial form [22]

$$
\begin{aligned}
\tilde{c}_{i j k l}= & \tilde{c}_{1} \delta_{i j} \delta_{k l}+\tilde{c}_{2}\left(\delta_{i k} \delta_{j l}+\delta_{i l} \delta_{k j}\right) \\
& +\tilde{c}_{3} M_{i} M_{j} M_{k} M_{l}+\tilde{c}_{4}\left(M_{i} M_{j} \delta_{k l}+M_{k} M_{l} \delta_{i j}\right) \\
& +\tilde{c}_{5}\left(M_{i} M_{k} \delta_{j l}+M_{i} M_{l} \delta_{j k}+M_{j} M_{k} \delta_{i l}+M_{j} M_{l} \delta_{i k}\right),
\end{aligned}
$$

$$
\begin{aligned}
\gamma_{i j k l}= & \gamma_{1} \delta_{i j} \delta_{k l}+\gamma_{2}\left(\delta_{i k} \delta_{j l}+\delta_{i l} \delta_{k j}\right) \\
& +\gamma_{3} M_{i} M_{j} M_{k} M_{l}+\gamma_{4} M_{i} M_{j} \delta_{k l}+\gamma_{5} M_{k} M_{l} \delta_{i j} \\
& +\gamma_{6}\left(M_{i} M_{k} \delta_{j l}+M_{i} M_{l} \delta_{j k}+M_{j} M_{k} \delta_{i l}+M_{j} M_{l} \delta_{i k}\right),
\end{aligned}
$$

where the first lines are the isotropic parts.

For the (isotropic) elastic moduli $\tilde{c}_{1}, \tilde{c}_{2}$, and $\tilde{\chi}_{u}$ we assume here that they are proportional to $M^{2}$ :

$$
\begin{gathered}
\tilde{c}_{n}=M^{2} c_{n} \quad \text { for } n \in\{1,2\}, \\
\tilde{\chi}_{u}=M^{2} \chi_{u} .
\end{gathered}
$$

This ensures that the elastic stress vanishes, when there are no columns, i.e., when the magnetization $\boldsymbol{M}$ is zero. This quadratic dependence is the simplest assumption, but can be replaced by a more complicated (even discontinuous) function $f_{2}\left(M^{2}\right)$ provided $f_{2}\left(M^{2}\right) \rightarrow 0$ for $M \rightarrow 0$. The dependence on $M^{2}$ (rather than $M_{i}$ ) is due to the time-reversal behavior of $\boldsymbol{M}$. The elastic coefficients $\tilde{c}_{3,4,5}$, describing elastic anisotropy due to the magnetic field, can be taken as constants, since they already come with a field dependence in Eq. (8), either quadratic $\left(\sim \tilde{c}_{4}\right.$ and $\left.\tilde{c}_{5}\right)$ or quartic $\left(\sim \tilde{c}_{3}\right)$, and therefore do not contribute in the absence of a field. We have kept the contribution $\sim \tilde{c}_{3}$ [and $\gamma_{3}$ in Eq. (7)]-although they are of fourth order in $\boldsymbol{M}$ - to reflect the complete structure of the tensors for uniaxial symmetry.

In a macroscopic description the actual values of the elastic moduli and coupling coefficients are not known a priori, but can be obtained from experimental results or mesoscopic simulations. Those coefficients reflect summarily the magnetic interactions of the individual magnetic particles in a mesoscopic description.

In the absence of the magnetic field the equilibrium magnetization is zero, $M_{i}=0$, and there is no strain, $U_{i j}=0$, and no concentration variation $\bar{\phi} \equiv \phi-\rho_{2} / \rho=0$. Then Eq. (5) gives the energetic cost for deviations from the equilibrium state. For a finite external field the new ground state is given by a finite magnetization due to the direct coupling $\sim H_{i} M_{i}$, by a nonzero strain $U_{k k}$ due to magnetostriction, and by a finite $\bar{\phi}$ due to the $\chi_{m}$ coupling. We will discuss the implication of this new ground state for the statics in detail in Appendix D.

Neglecting the probably small magnetic-field-induced strains and concentration shift in the ground state, we can use Eq. (5) as the hydrodynamic energy and get for the conjugate quantities

$$
\begin{aligned}
& h_{i}^{M}=\frac{\delta \varepsilon}{\delta M_{i}}=\left(\alpha+\beta M^{2}\right) M_{i}-\mu_{0} H_{i}+2 \chi_{u} U_{k k} \bar{\phi} M_{i} \\
&-\frac{1}{2} \gamma_{k j l i}^{i s o}\left(U_{k j} M_{l}+U_{l j} M_{k}\right)+2 \chi_{m} \bar{\phi} M_{i} \\
&+c_{p j k l}^{i s o} U_{p j} U_{k l} M_{i}, \\
& \Phi_{i j}=\frac{\delta \varepsilon}{\delta U_{i j}}=c_{i j k l} U_{k l} M^{2}-\frac{1}{2} \gamma_{i j k l} M_{k} M_{l}+\chi_{u} \delta_{i j} M^{2} \bar{\phi}, \\
& \delta \Pi=\frac{\delta \varepsilon}{\delta \bar{\phi}}=\chi_{\phi} \bar{\phi}+\chi_{u} M^{2} U_{k k}+\chi_{m} M^{2},
\end{aligned}
$$

where $\delta \Pi$ is the excess osmotic pressure beyond its equilibrium value $\rho\left(\mu_{1}-\bar{\mu}_{2}\right)$ [see Eq. (A7)]. In Eq. (10) we show 
only the isotropic parts of the elastic and magnetostrictive tensors [first lines of Eqs. (8) and 7)], since the anisotropic parts have a more complicated dependence on $M_{i}$.

\section{B. Macroscopic dynamics}

The dynamic evolution of deviations from the equilibrium state is described by the appropriate macroscopic equations for the variables introduced above. Since we simplify the model by assigning proper transport velocities to the variables, the general equations are specialized by giving certain values to some (reversible) transport parameters. This is done and explained in Appendix D and the final expressions are

$$
\begin{gathered}
\dot{\rho}+\nabla_{j}\left(\rho v_{j}\right)=0 \\
\dot{\phi}+v_{j}^{(c)} \nabla_{j} \phi+\rho^{-1}\left(\phi[1-\phi] \nabla_{i} \rho w_{i}+\nabla_{i} j_{i}^{\phi}\right)=0 \\
\dot{\varepsilon}+\nabla_{j}(\varepsilon+p) v_{j}+\nabla_{i} j_{i}^{\varepsilon}=0 \\
\dot{\sigma}+\nabla_{j}\left(\sigma v_{j}+j_{i}^{\sigma}\right)=2 R / T \\
\dot{w}_{i}+v_{j}^{(c)} \nabla_{j} w_{i}+\nabla_{i} \Pi+\nabla_{j}\left(\rho_{2}^{-1} \Phi_{i j}\right)+w_{j} \nabla_{j} v_{i} \\
-\left(\rho_{2}-\rho_{1}\right) \rho^{-1} w_{j} \nabla_{i} w_{j}+\rho_{2}^{-1} \Phi_{k j} \nabla_{i} U_{k j} \\
-2 \nabla_{j}\left(\rho_{2}^{-1} \Phi_{k j} U_{i k}\right)+\rho_{2}^{-1} h_{j}^{M} \nabla_{i} M_{j} \\
+\rho_{2}^{-1} \nabla_{k}\left(h_{i}^{M} M_{k}-h_{k}^{M} M_{i}\right)+Y_{i}=0 \\
\dot{g}_{i}+\nabla_{j}\left(g_{i} v_{j}+h_{i}^{w} w_{j}\right)+\nabla_{j}\left(-\Phi_{i j}+\Phi_{j k} U_{i k}+\Phi_{i k} U_{j k}\right) \\
+\nabla_{i} p-\frac{1}{2} \nabla_{j}\left(B_{i} H_{j}+B_{j} H_{i}\right)+\nabla_{j} \sigma_{i j}=0 \\
\dot{M}_{i}+v_{j}^{(2)} \nabla_{j} M_{i}+\epsilon_{i j k} M_{j} \omega_{k}^{(2)}+X_{i}=0 \\
\dot{U}_{i j}+v_{k}^{(2)} \nabla_{k} U_{i j}+U_{k j} \nabla_{i} v_{k}^{(2)}+U_{k i} \nabla_{j} v_{k}^{(2)}-A_{i j}^{(2)}+Z_{i j}=0
\end{gathered}
$$

where $v_{i}^{(c)} \equiv \phi v_{i}^{(2)}+(1-\phi) v_{i}^{(1)}$ is the convective velocity for $\phi$ and $w_{i}$, while $A_{i j}^{(2)}=(1 / 2)\left(\nabla_{i} v_{j}^{(2)}+\nabla_{j} v_{i}^{(2)}\right)$ is the symmetric gradient of the second velocity and the vorticity $2 \omega_{i}^{(2)}=\epsilon_{i j k} \nabla_{j} v_{k}^{(2)}$ corresponds to its antisymmetric gradient. Note that the phenomenological currents (e.g. $j_{i}^{\phi}, Y_{i}, \sigma_{i j}, X_{i}$, $Z_{i j}$ ) do not contain the contributions of Eqs. (B9)-(B13), since the latter are already contained in the dynamic equations, above.

There is no independent conservation law for the total angular momentum, since the latter is not the sum of the local angular momenta of the individual particles [23]. Instead the total orbital angular momentum is nonlocal [23]. As a result angular momentum requires the antisymmetric part of the stress tensor $\sigma_{i j}$ to be zero or a pure divergence [24].

The form of Eqs. (13) and (14) guarantees that the densities $\rho_{1,2}$ are transported by $\boldsymbol{v}_{1,2}$, respectively, as shown in Eqs. (B14) and (B15). Similarly, Eqs. (17) and (18) lead to the desired form of the dynamics of $\boldsymbol{g}_{1,2}$ [see Eqs. (B16) and (B17)]. The nonphenomenological parts of the currents shown explicitly are not related to any phenomenological (transport) parameters and are given by general symmetry and thermodynamic principles [20]. We emphasize that their structure, in particular that of the convective derivative $U_{k j} \nabla_{i} v_{k}+U_{k i} \nabla_{j} v_{k}$ in Eq. (20), is uniquely determined [25-27], also in the two-fluids case [17,19]. The flow term, $-A_{i j}^{(2)}$ in Eq. (20), guarantees translational invariance of that equation, and the current $Z_{i j}$ must not contain any $A_{i j}$ contribution.

The set of dynamic equations contains five phenomenological currents (disregarding the entropy current), in particular, the stress tensor $\sigma_{i j}$, the concentration current $j_{i}^{\phi}$, and the quasicurrents $X_{i}, Y_{i}$, and $Z_{i j}$ that describe temporal changes of their corresponding variables. They are functions of the thermodynamic forces introduced via Eq. (1) and involve phenomenological transport parameters. They can be written as a sum of a reversible (superscript $R$ ) and an irreversible part (superscript $D$ ). Contributions are reversible, when they transform under time reversal, $t \rightarrow-t$, in the same way as the time derivative of their appropriate variable, while dissipative ones transform oppositely. For a general discussion of time-reversal symmetry and its importance for macroscopic equations we refer to Ref. [28].

The second law of thermodynamics states that irreversible dynamic processes always dissipate energy (transfer energy to the microscopic degrees of freedom as heat) and therefore increase the entropy. In contrast, reversible processes are nondissipative and must not increase the entropy.

Within linear irreversible thermodynamics [29] the dissipation function $R$, which is proportional to the entropy production, can be written as a bilinear form of fluxes and forces:

$$
2 R=-j_{i}^{\phi} \nabla_{i} \Pi-\sigma_{i j} A_{i j}+X_{i} h_{i}^{M}+Z_{i j} \Phi_{i j}+Y_{i} h_{i}^{w},
$$

which is zero, $R=0$, for reversible currents and is positive, $R>0$, for irreversible ones.

To derive the dissipative parts of the (quasi-)currents one writes the dissipation function $R$ as a quadratic form in the relevant thermodynamic forces. By taking the variational derivative of this function with respect to the chosen thermodynamic force, according to Eq. (21), one gets the corresponding dissipative current. The dissipation function is

$$
\begin{aligned}
R= & \frac{1}{2} v_{i j k l}^{D} A_{i j} A_{k l}+\frac{1}{2} b_{i j}^{D} h_{i}^{M} h_{j}^{M}+\frac{1}{2} D_{i j}\left(\nabla_{i} \Pi\right)\left(\nabla_{j} \Pi\right) \\
& +\frac{1}{2}(1 / \tau)_{i j k l} \Phi_{i j} \Phi_{k l}+d_{i j k} \Phi_{j k} h_{i}^{M}+\frac{1}{2} \xi_{i j} h_{i}^{w} h_{j}^{w} \\
& +\frac{1}{2} v_{i j k l}^{(w)}\left(\nabla_{j} h_{i}^{w}\right)\left(\nabla_{l} h_{k}^{w}\right)+v_{i j k l}^{(c)} A_{i j} \nabla_{l} h_{k}^{w} \\
& +\alpha_{i j k}\left(\nabla_{j} \Pi\right)\left(\nabla_{k} h_{i}^{M}\right)+\beta_{i j k} A_{i j} h_{k}^{M}+\gamma_{i j k}\left(\nabla_{i} h_{j}^{w}\right) h_{k}^{M} \\
& +\frac{1}{2} r_{i j k l}\left(\nabla_{j} h_{i}^{M}\right)\left(\nabla_{l} h_{k}^{M}\right) .
\end{aligned}
$$

Magnetic relaxation $b_{i j}^{D}$, relative velocity relaxation $\xi_{i j}$, and diffusion $D_{i j}$ are isotropic in lowest order of $M^{2}$, and uniaxial in general,

$$
l_{i j}=l \delta_{i j}+l_{\|} M_{i} M_{j},
$$

for $l \in\left\{b^{D}, \xi, D\right\}$.

The fourth rank tensors $v_{i j k l}^{D}, v_{i j k l}^{(w)}$, and $r_{i j k l}$ are of the same form as $\tilde{c}_{i j k l}$, Eq. (8), while $v_{i j k l}^{(c)}$ is as $\gamma_{i j k l}$, Eq. (7). We note that the contribution $\sim r_{i j k l}$ is a higher order gradient term 
compared to the term $\sim b_{i j}^{D}$; it has been kept to guarantee positivity of the entropy production.

The elastic relaxation tensor $(1 / \tau)_{i j k l}$ has the same tensorial form as the elastic moduli tensor, including the $M^{2}$ dependence of the coefficients, and is isotropic in lowest order of $M^{2}$,

$$
(1 / \tau)_{i j k l}=\frac{1}{\tau_{1} M^{2}} \delta_{i j} \delta_{k l}+\frac{1}{\tau_{2} M^{2}}\left(\delta_{i k} \delta_{j l}+\delta_{j k} \delta_{i l}\right),
$$

while the general uniaxial form is given by Eq. (8), when $\tilde{c}_{n}$ is replaced by $1 /\left(\tau_{n} M^{2}\right)$.

Thus, we model the viscoelastic properties of MRFs [30-36] by using the strain field as a relaxing variable. When the columns are deformed, the particles experience a drive to redistribute, e.g., by permeation effects, which shows on the macroscopic level as relaxation of the strain. In addition, we assume that the relaxation coefficients, $\tau_{1} M^{2}$ and $\tau_{2} M^{2}$, are proportional to $M^{2}$, which is motivated by the experimental fact that the elastic network relaxes on longer time scales in larger magnetic fields, i.e., it behaves more elastically [31]. We discard elastic diffusion-type contributions $\sim \nabla_{k} \Phi_{i j}$.

In Eq. (22), the third rank material tensors come in two classes. First, $d_{i j k}$, representing the dissipative coupling of the elastic stress to the magnetization, and $\alpha_{i j k}$, relating concentration gradients with inhomogeneous magnetization, have to be odd in $M_{i}$ and both are symmetric in $j, k$ and read with $l \in\{d, \alpha\}$

$$
l_{i j k}=l_{1} M_{i} \delta_{j k}+l_{2}\left(M_{j} \delta_{i k}+M_{k} \delta_{i j}\right)+l_{3} M_{i} M_{j} M_{k}
$$

The dissipative third rank tensors $\beta_{i j k}$ and $\gamma_{i j k}$ have to be even in $M_{i}$ in order to have the correct time-reversal behavior. First,

$$
\beta_{i j k}=\beta\left(M_{i} \epsilon_{j k l}+M_{j} \epsilon_{i k l}\right) M_{l},
$$

relating magnetization dynamics to mean flow, is quadratic in the magnetization and of a similar structure as the tensor $c_{i j k}^{J}$ given for systems with an axial dynamic preferred direction in Ref. [37], when $w_{i}$ in Ref. [37] is replaced by $M_{i}$. It has been introduced to ferrofluids with one velocity field in Ref. [38] where $\beta$ is called $\lambda_{4} / 2$.

The tensor $\gamma_{i j k}$, relating magnetization dynamics to relative velocities presented here,

$$
\gamma_{i j k}=\gamma_{1}^{D} \epsilon_{i j k}+\gamma_{2}^{D} \epsilon_{i j l} M_{k} M_{l}+\gamma_{3}^{D} \epsilon_{i k l} M_{j} M_{l}+\gamma_{4}^{D} \epsilon_{j k l} M_{i} M_{l} \text {, }
$$

does not have any symmetry and contains generally four coefficients, of which one is still present in the isotropic approximation.

Taking variational derivatives of Eq. (22) we have for the dissipative current and quasicurrents

$$
\begin{gathered}
\sigma_{i j}^{D}=-(\partial R) /\left(\partial A_{i j}\right)=-v_{i j k l}^{D} A_{k l}-v_{i j k l}^{(c)} \nabla_{l} h_{k}^{w}-\beta_{i j k} h_{k}^{M} \\
Z_{i j}^{D}=(\partial R) /\left(\partial \Phi_{i j}\right)=(1 / \tau)_{i j k l} \Phi_{k l}+d_{k i j} h_{k}^{M} \\
Y_{i}^{D}=(\partial R) /\left(\partial h_{i}^{w}\right)= \\
\xi_{i j} h_{j}^{w}-\nabla_{j}\left(\gamma_{j i k} h_{k}^{M}\right) \\
-\nabla_{j}\left(v_{i j k l}^{(w)} \nabla_{l} h_{k}^{w}+v_{i j k l}^{(c)} A_{k l}\right)
\end{gathered}
$$

$$
\begin{aligned}
& X_{i}^{D}=(\partial R) /\left(\partial h_{i}^{M}\right)= b_{i j}^{D} h_{j}^{M}+d_{i j k} \Phi_{j k}+\beta_{k j i} A_{k j} \\
&+\gamma_{j k i}\left(\nabla_{k} h_{j}^{w}\right)-\nabla_{k}\left(\alpha_{i j k} \nabla_{j} \Pi\right) \\
&-\nabla_{j}\left(r_{i j k l} \nabla_{l} h_{k}^{M}\right), \\
& j_{i}^{\phi D}=-(\partial R) /\left(\partial \nabla_{i} \Pi\right)=-D_{i j} \nabla_{j} \Pi-\alpha_{j i k} \nabla_{k} h_{j}^{M} .
\end{aligned}
$$

The reversible currents cannot be derived from the dissipation function, since $R \equiv 0$ for the reversible case. Instead, they are set up by collecting all possible combinations allowed by (e.g., time-reversal) symmetry that lead to a vanishing $R$ in Eq. (21). In Appendix D we have discussed all reversible couplings related to transport (advection and convection), Eqs. (B9)-(B13), and assigned values to the material parameters such that variables of group 1 (2) are transported by the velocity $\boldsymbol{v}_{1}\left(\boldsymbol{v}_{2}\right)$. Those contributions are already built in in Eqs. (14)-(20), and here we show the remaining phenomenological reversible currents:

$$
\begin{gathered}
j_{i}^{\phi R}=b_{i j}^{\phi} \nabla_{j} \Pi, \\
Y_{i}^{R}=\xi_{i j k} \nabla_{j} h_{k}^{M}, \\
X_{i}^{R}=b_{i j}^{R} h_{j}^{M}-c_{i j k}^{R} A_{j k}+\zeta_{i j k} \Phi_{j k}+\xi_{k j i} \nabla_{j} h_{k}^{w}, \\
\sigma_{i j}^{R}=-v_{i j k l}^{R} A_{k l}-c_{k i j}^{R} h_{k}^{M}, \\
Z_{i j}^{R}=-\zeta_{k i j} h_{k}^{M}+v_{i j k l}^{u} \Phi_{k l} .
\end{gathered}
$$

The coupling between relative velocity and magnetization, $\sim \xi_{i j k}$, has to be odd in $M_{i}$ in order to be reversible:

$$
\xi_{i j k}=\xi_{1} M_{i} \delta_{j k}+\xi_{2} M_{j} \delta_{i k}+\xi_{3} M_{k} \delta_{i j}+\xi_{4} M_{i} M_{j} M_{k} .
$$

The tensor $\zeta_{i j k}$ is even in $M_{i}$ and has the same form as $\beta_{i j k}$ in Eq. (26) with $\beta$ replaced by $\zeta$. A similar coupling is found in Eq. (33) of Ref. [37], when $w_{i}$ in Ref. [37] is replaced by the magnetization $M_{i}$. The tensor $c_{i j k}^{R}$ is of the form Eq. (25) with $l \equiv c^{R}$. It has been introduced to ferrofluid hydrodynamics with one velocity field in Ref. [38] with the correspondences $c_{1}^{R}=>-\lambda_{1}+(1 / 3)\left(\lambda_{2}+\lambda_{3} M_{0}^{2}\right), c_{2}^{R}=>-\lambda_{2} / 2$, and $c_{3}^{R}=>-\lambda_{3}$. The contribution $\sim c_{2}^{R}$ corresponds to the flow alignment coefficient in nematic liquid crystals.

In Eqs. (25) and (38) we have kept contributions cubic in $\boldsymbol{M}$ in the same spirit as in Ref. [21]. The reversible contributions without a counterterm $b_{i j}^{R, \phi}$ are of the form

$$
b_{i j}^{R}=b^{R} \epsilon_{i j k} M_{k} \quad \text { and } \quad b_{i j}^{\phi}=b^{\phi} \epsilon_{i j k} M_{k}
$$

while $v_{i j k l}^{R, u}$ is as given by

$$
\begin{aligned}
v_{i j k l}^{R, u}= & v_{1}^{R, u}\left(\epsilon_{i k p} \delta_{j l}+\epsilon_{j k p} \delta_{i l}+\epsilon_{i l p} \delta_{j k}+\epsilon_{j l p} \delta_{i k}\right) M_{p} \\
& +v_{2}^{R, u}\left(\epsilon_{i k p} M_{j} M_{l}+\epsilon_{j k p} M_{i} M_{l}+\epsilon_{i l p} M_{j} M_{k}\right. \\
& \left.+\epsilon_{j l p} M_{i} M_{k}\right) M_{p} .
\end{aligned}
$$

Due to their antisymmetric structure, $b_{i j}^{R, \phi}=-b_{j i}^{R, \phi}$ and $v_{i j k l}^{R, u}=-v_{k l i j}^{R, u}$, those contributions lead to zero entropy production. To make these currents reversible, the tensors have to be odd functions in $M_{i}$. 


\section{SUGGESTIONS FOR EXPERIMENTS ON MRF}

\section{A. General remarks}

It is instructive to compare the two-fluid model of magnetorheological fluids with the recently derived one-fluid model [18]. The one-fluid model is a special limiting case of the two-fluid one. For a detailed overview of how the reduction of the two-fluid model to the one-fluid model is done, we refer the reader to Appendix E. Obviously, the velocity difference and the concentration are the specific two-fluid variables. In this section we discuss the linear couplings of these variables with the magnetization. In particular, we show how a magnetic gradient field can induce a nonzero relative velocity. In Sec. III B two-fluid effects on the elastic degree of freedom are considered, describing a first step towards column formation. In Sec. III C we deal with two-fluid effects regarding flow properties.

In the statics we get the conjugates of the magnetization and the concentration in terms of linear deviations from the ground state [Appendix D, Eqs. (D8) and (D10)],

$$
\begin{aligned}
& h_{i}^{M}=\frac{\delta \varepsilon}{\delta M_{i}}=\alpha \delta M_{i}+2 \chi_{m} M_{i}^{0} \delta \phi, \\
& \delta \Pi=\frac{\delta \varepsilon}{\delta \phi}=\chi_{\phi} \delta \phi+2 \chi_{m} M_{i}^{0} \delta M_{i},
\end{aligned}
$$

and there are no static cross couplings with velocity differences in our model, $h_{i}^{w}=\left(\rho_{1} \rho_{2} / \rho\right) w_{i}$.

For the linearized dynamics of the considered variables, Eqs. (14), (17), and (19) can be written as

$$
\begin{gathered}
\rho \dot{\phi}+\nabla_{i} h_{i}^{w}+\nabla_{i} j_{i}^{\phi}=0, \\
\dot{w}_{i}+\nabla_{i} \Pi+Y_{i}=0, \\
\dot{M}_{i}+X_{i}=0,
\end{gathered}
$$

where the phenomenological currents, Eqs. (30)-(35), show the mutual cross couplings involving gradients of $h_{i}^{w}$ and gradients of $h_{i}^{M}$. These couplings are described by the dissipative material tensors, $\gamma_{i j k}$ and $\alpha_{i j k}$, and a reversible one $\xi_{i j k}$. Gradients of $h_{i}^{M}$ can be realized by adding a small inhomogeneous external magnetic field (to the main homogeneous field that is responsible for $M_{0}$ ). For stationary situations and with the field gradient transverse to the field direction, $\nabla_{j} H_{i}$ can induce a finite transverse relative velocity. In lowest order of $M_{0}$,

$$
w_{i} \sim\left(-\gamma_{1}^{D} \epsilon_{i j z}+\left[\alpha_{1} D^{-1}-\xi_{3}\right] M_{0} \delta_{i j}^{\perp}\right) \mu_{0} \nabla_{j} H_{z},
$$

and $w_{i}$ is rotated in the transverse plane with respect to the gradient direction by an angle $\vartheta$, with $\tan \vartheta=\left(\alpha_{1} D^{-1}-\right.$ $\left.\xi_{3}\right) M_{0} / \gamma_{1}^{D}$. One can therefore tune this angle by the homogeneous part of the field, which sets $M_{0}$. Apart from the direct couplings $\sim \gamma_{1}^{D}$ and $\sim \xi_{3}$ according to Eqs. (30) and (34), there is an indirect coupling $\sim \alpha_{1}$ due to the fact that constant field gradients drive stationary concentration gradients, $\nabla_{j} \Pi=-D_{i j}^{-1} \alpha_{k i l} \nabla_{l} h_{k}^{M}$, according to Eq. (32). The latter relation also leads to interesting effects on the elastic properties, in particular the yield stress in MRFs, when the gradient of the field is along the field direction.

\section{B. Influence of spatial modulations}

One of the outstanding features of MRFs and ERFs is the formation of columnar structures as an external field (magnetic for MRFs or electric for ERFs) is applied and increased (see Ref. [39]). The phenomena leading to the formation of columns as a function of increasing field strength can be summarized as follows. For low fields small clusters are formed in parallel with the existence of individual particles similar to the case of magnetic fluids [40]. As the field is increased the chains and clusters become longer and eventually they become long enough to connect the two bounding plates of a sample, while shorter chains still exist in parallel. As the field is increased even further the chains eventually start to merge to form a columnar structure of ideally hexagonal symmetry.

It appears important to notice that there are apparently very few quantitative studies of the question of how physical properties are affected by this gradual formation of chains and columns as a function of an applied field. For MRFs we would like to mention two studies. In Ref. [41] the linear viscoelasticity as a function of applied magnetic field has been investigated. A continuous variation of the storage modulus $G^{\prime}$ and of the loss tangent, $\tan \delta$, as a function of the external magnetic field is found until column formation is essentially finished. In Ref. [42] the static and dynamic yield stress has been investigated and in both cases smooth curves as a function of the external magnetic field were found until column formation was completed. We finally mention an experiment on the dielectric investigation of chain formation in ERFs [43]. A continuous change in dielectric permittivity as a function of electric field strength was observed. In addition, a break in slope of this quantity was described in the vicinity of the crossover from single chains to columns as a function of field strength.

Here we will analyze as a first step transverse spatial modulations as a function of magnetic field for MRFs, focusing on small amplitude spatial modulations due to an external magnetic field around a spatially homogeneous situation. We will demonstrate that even this first step leads to segregation, which - by construction - cannot be achieved in a one-fluid model. We study the combined dynamics of $w_{i}, M_{i}, \phi$, and the strain field $U_{i j}$, and discuss spatial modulations due to an external field. We find that the relative velocity plays an important role to explain this phenomenon. We use the linearized equations shown above and make some additional assumptions to make the problem tractable. The magnetic field is along the $z$ axis and in the ground state the magnetization, $M_{0}$, is homogeneous and parallel to the field. We assume the fluctuations are homogeneous in the $z$ direction, i.e. all gradients $\nabla_{z}=0$. In that case we are left with five variables, $\delta \phi, \delta M_{z}$, and $\nabla \cdot \boldsymbol{w}$, and the strains $U_{z z}$ and $U_{k k}$. Finally we consider the time range where the magnetization, the velocity differences, and the strains are already relaxed and only temporal changes of the concentration are present. With these assumptions we get for transverse wavelike fluctuations $f=$ $\tilde{f} e^{\mathrm{i} k_{x} x+\mathrm{i} k_{y} y} e^{\sigma t}$ of the relevant variables the linearized dynamic equations

$$
\begin{gathered}
\rho \sigma \delta \phi+D k_{\perp}^{2} \Pi+\nabla \cdot \boldsymbol{h}^{w}+\alpha_{1} M_{0} k_{\perp}^{2} h_{z}^{M}=0, \\
k_{\perp}^{2} \Pi-\xi \nabla \cdot \boldsymbol{h}^{w}+\xi_{3} M_{0} k_{\perp}^{2} h_{z}^{M}=0,
\end{gathered}
$$




$$
\begin{gathered}
\alpha_{1} M_{0} k_{\perp}^{2} \Pi+\xi_{3} M_{0} \nabla \cdot h^{w}+b^{D} h_{z}^{M}=0, \\
\frac{1}{\tau_{1}} \delta_{i j} \Phi_{k k}+\frac{2}{\tau_{2}} \Phi_{i j}+\left(d_{1} \delta_{i j}+2 d_{2} \delta_{i z} \delta_{j z}\right) M_{0} h_{z}^{M}=0,
\end{gathered}
$$

where we have neglected the $d_{3} M_{0}^{2}$ contribution for simplicity. The dissipative cross coupling due to $\gamma_{i j k}$ and the reversible one due to $\zeta_{i j k}$ are not in effect in this geometry.

The variable $\delta \phi$ can be expressed linearly by the thermodynamic forces using the relations Eqs. (D8)-(D10). Solving the resulting linearized equations one gets for the relaxation or growth rate, $\sigma$, of the fluctuations

$$
\frac{\rho \sigma}{M_{\text {sus. }}}=k_{\perp}^{2} \frac{P_{0}+P_{1} M_{0}^{2}+P_{2} k_{\perp}^{2}+P_{3} M_{0}^{2} k_{\perp}^{2}}{N_{0}+N_{1} M_{0}^{2}+N_{2} k_{\perp}^{2}+N_{3} M_{0}^{2} k_{\perp}^{2}},
$$

where $M_{\text {sus. }}$ is the determinant of the static susceptibility matrix, which is always positive for stability reasons. The field-independent abbreviations $P_{0,1,2,3}$ and $N_{0,1,2,3}$ contain rather complicated combinations of static susceptibilities and dynamic transport coefficients. This rather simple form has been obtained by neglecting contributions $O\left(M_{0}^{2}\right)$ in Eqs. (D2) and (D3). These coefficients are in general not known and have to be determined either from an experiment or from a microscopic simulation of the system. By measuring the rate of the fluctuations, described by Eq. (51), as a function of the wave vector perpendicular to the magnetic field $k_{\perp}$, then one could get an idea of the values of the coefficients $N_{0,1,2,3}$ and $P_{0,1,2,3}$.

Equation (51) already allows for some basic insights into the formation of spatial patterns in MRFs. We observe immediately that in the model presented this rate is invariant with respect to a rotation of the wave vector around the $z$ axis, which corresponds to the fact that there is no preferred direction in the plane perpendicular to the external magnetic field.

Maximizing the growth rate $\sigma$ given in Eq. (51) with respect to $k_{\perp}$ one obtains a quadratic polynomial in $k_{\perp}^{2}$. Inserting the critical value $k_{\perp}^{c}$ into $\sigma$ leads to a critical $\sigma_{c}$ that is a complicated function of $M_{0}^{2}$, which generally allows for a finite threshold, where $\sigma_{c}$ changes sign, indicating the onset of the formation of spatial patterns.

It should be noted that if one neglects the dynamic couplings, $d_{1}$ and $d_{2}$, between the elastic degree of freedom and the magnetization, and the magnetic diffusion, $r_{2}$, one gets $P_{1,2}=0=N_{1,2}$, resulting in $k_{\perp}^{c} \sim M_{0}^{-1}$ and $\sigma_{c} \sim M_{0}^{-2}$ without any threshold for pattern formation. In this special case the remaining nonzero abbreviations in Eq. (51) read

$$
\begin{gathered}
P_{0}=-b^{D}(1+\xi D), \\
P_{3}=\alpha_{1}^{2} \xi-D \xi_{3}^{2}+2 \alpha_{1} \xi_{3}, \\
N_{0}=\alpha b^{D} \xi, \\
N_{3}=2 \chi_{m} \alpha_{1} \xi+\alpha \xi_{3}^{2}+2 \chi_{m} \xi_{3}, \\
M_{\text {sus. }}=\alpha \chi_{\phi}-4 \chi_{m}^{2} M_{0}^{2} .
\end{gathered}
$$

It seems that so far no publications on modeling pattern formation using a hydrodynamic description, for example, the transition of columnar structures to lamellae in MRFs, have appeared. In a future paper we will therefore analyze pattern formation and instabilities in MRF using the two-fluid description given here [44]. We note in this context that MRFs have the advantage of not having space charge effects and there is no analog of the electric conductivity of ERFs.

\section{Two-fluid flow properties}

In Ref. [18] much emphasis was laid on the MRF behavior under external static strains and stationary and oscillatory shear flow in the presence of a magnetic field. Therefore, we discuss here the coupling of the specific two-fluid variables, $w_{i}$ and $\bar{\phi}$, to flow and to the elastic degrees of freedom.

In the statics, $w_{i}$ does not couple to other variables, but $\bar{\phi}$ does. For the one-fluid case, by setting the excess osmotic pressure Eq. (12) to zero, $\bar{\phi}$ is expressed by strains and the magnetic field and can be replaced in Eqs. (10) and (11). As a result, the appropriate linear one-fluid equations are the same as the two-fluid ones, when the replacements

$$
\begin{gathered}
\gamma_{1} \rightarrow \gamma_{1}-2 \chi_{m} \chi_{u} \chi_{\phi}^{-1} M_{0}^{2}, \\
\beta \rightarrow \beta-2 \chi_{m}^{2} \chi_{\phi}^{-1}, \\
c_{1} \rightarrow c_{1}-\chi_{u}^{2} \chi_{\phi}^{-1} M_{0}^{2}
\end{gathered}
$$

are made. Therefore the statics of the one-fluid description is, neglecting the anisotropic structure of the tensors $c_{i j k l}$ and $\gamma_{i j k l}$, isomorphic to the statics of the two-fluid description.

In the dynamics, relative velocities couple to flow through the dissipative tensor $v_{i j k l}^{(c)}$ in Eqs. (28) and (30):

$$
\begin{aligned}
\sigma_{i j}^{D} & =-v_{i j k l}^{(c)} \nabla_{l} h_{k}^{w}+\ldots, \\
Y_{i}^{D} & =-v_{i j k l}^{(c)} \nabla_{j} A_{k l}+\ldots,
\end{aligned}
$$

meaning gradients of $h_{i}^{w}$ (and therefore gradients of $w_{i}$ ) couple to the stress tensor. Thus, there are two different viscosities involved,

$$
\begin{aligned}
\sigma_{i j}^{D} & =-v_{i j k l}^{D} A_{k l}-v_{i j k l}^{(c)}\left(\rho_{1} \rho_{2} / \rho\right)\left(\nabla_{k} w_{l}+\nabla_{l} w_{k}\right) \\
& =-v_{i j k l}^{(1)} A_{k l}^{(1)}-v_{i j k l}^{(2)} A_{k l}^{(2)},
\end{aligned}
$$

either related to gradients of $v_{i}$ and $w_{i}$ or to $v_{i}^{(1)}$ and $v_{i}^{(2)}$. It is a key aspect of our two-fluid model that the magnetization and the magnetic particles are transported with different velocities giving rise to two different viscosities. It is not possible to account for such a behavior in the one-fluid model, where both the carrier fluid and the particulate phase are transported with the suspension velocity.

There are cross couplings between flow and magnetization [see Eqs. (28) and (31) for the dissipative part, and Eqs. (36) and (35) for the reversible part], provided by the material tensors $\beta_{i j k}$ and $c_{i j k}^{R}$, respectively. If shear flow is applied, stationary stresses are induced:

$$
\begin{gathered}
\sigma_{x z}=-2 v_{2}^{\text {eff }} A_{x z}-2 v_{1}^{R, \text { eff }} M_{0} A_{y z}, \\
\sigma_{y z}=-2 v_{2}^{\text {eff }} A_{y z}+2 v_{1}^{R, \text { eff }} M_{0} A_{x z},
\end{gathered}
$$

with the field dependent effective shear viscosity

$$
v_{2}^{\text {eff }}=v_{2}+\left(c_{2}^{R}\right)^{2} \frac{b^{D} M_{0}^{2}}{\left(b^{D}\right)^{2}+\left(b^{R}\right)^{2} M_{0}^{2}}
$$


and the effective reversible transport parameter

$$
v_{1}^{R, \text { eff }}=v_{1}^{R}+c_{2}^{R} M_{0}^{2} \frac{2 b^{D} \beta-b^{R} c_{2}^{R}}{\left(b^{D}\right)^{2}+\left(b^{R}\right)^{2} M_{0}^{2}}
$$

that describes stresses in a plane perpendicular to the shear flow plane.

Next we discuss the stationary equation for the strain field. In Ref. [18] it was found that the relaxing dynamics of the strain field is one of the key ingredients to model the flow curves, i.e., the dependence of the shear stress on the shear rate. In the one-fluid model the strain field is then induced by the shear rate of the suspension. We notice that in the present model the strain field is induced by the gradients of the velocity of the particulate phase $A_{i j}^{(2)}$ [see Eq. (20)]. This gradient can then be decomposed into symmetric gradients of the mean velocity and the relative velocity:

$$
A_{i j}^{(2)}=A_{i j}-\frac{\phi}{2}\left(\nabla_{i} w_{j}+\nabla_{j} w_{i}\right)-\frac{1}{2}\left(w_{i} \nabla_{j} \phi+w_{j} \nabla_{i} \phi\right),
$$

where the last term can be neglected if one is interested only in the lowest order.

Consequently, if one imposes a shear flow under the assumption of a linear shear flow profile of the mean velocity, $v_{x}=\Gamma_{x} z$, the induced strain field is, neglecting the tensor $d_{i j k}$ [see Eq. (20)],

$$
U_{x z}=\frac{\tau_{2}}{8 c_{2}}\left(\Gamma_{x}-\phi\left[\nabla_{z} w_{x}+\nabla_{x} w_{z}\right]\right) .
$$

However, assuming a linear shear profile in the suspension velocity $v_{x}^{(1)}=\Gamma_{x} z$, the induced strain,

$$
U_{x z}=\frac{\tau_{2}}{8 c_{2}}(1-2 \phi) \nabla_{z} v_{x}^{(2)}
$$

is not directly driven by $\Gamma_{x}$, but through the viscous coupling between gradients of $v_{x}^{(1)}$ and $v_{x}^{(2)}$.

However, neither $v_{x}=\Gamma_{x} z$, nor $v_{x}^{(1)}=\Gamma_{x} z$, nor any linear flow profile of the other velocities is compatible with a stationary state, except for $w_{x} \equiv 0$, which brings us back to the one-fluid case. The rather general question of shear flow (and the appropriate boundary conditions) has - to the best of our knowledge - never been addressed systematically for any two-fluid system, but goes beyond the scope of the present paper.

\section{COMPARISON WITH TWO-FLUID EFFECTS IN ELECTRORHEOLOGICAL FLUIDS}

For ERF, two-fluid effects have been discussed before. Von Pfeil et al. [45,46] have studied a dynamic equation for the particle concentration in ERF coupled to the particle flux relative to the mean suspension motion. However, dynamic equations for one or, in particular, two velocity field(s) have not been given in Refs. [45,46]. The authors used in the sequel their approach to study pattern formation in ERF using only the dynamic equation for the concentration. Correspondingly the authors were restricted in their analysis to stationary patterns, since for an oscillatory pattern to arise as a possibility more than one dynamic equation would be necessary. These studies focused on mesoscopic scales including studies of shear banding. Shear banding, lamellar structures, and microstructures have been investigated in ERFs, mainly experimentally in Refs. [47-49].

More recently Sheng and coworkers [50-52] set up a twofluid model for ERF containing two dynamic equations for two velocity fields, where $\boldsymbol{V}_{s}$ denotes the velocity of the dense colloidal phase and $\boldsymbol{V}_{l}$ denotes the velocity associated with the purely liquid fraction. The resulting equations take the form [see Eqs. (43) and (44) of Ref. [51]]

$$
\begin{gathered}
\rho_{s}\left(\frac{\partial \boldsymbol{V}_{s}}{\partial t}+\boldsymbol{V}_{s} \cdot \nabla \boldsymbol{V}_{s}\right)= \\
-\nabla p_{s}+\nabla \cdot \boldsymbol{\tau}_{\mathrm{visc}}^{s}+\nabla \cdot \boldsymbol{\tau}_{s} \\
+K\left(\boldsymbol{V}_{l}-\boldsymbol{V}_{s}\right), \\
\rho_{l}\left(\frac{\partial \boldsymbol{V}_{l}}{\partial t}+\boldsymbol{V}_{l} \cdot \nabla \boldsymbol{V}_{l}\right)=-\nabla p_{l}+\nabla \cdot \boldsymbol{\tau}_{\mathrm{visc}}^{l}+K\left(\boldsymbol{V}_{s}-\boldsymbol{V}_{l}\right) .
\end{gathered}
$$

These equations were derived together with a continuity equation for the density of solid particles using a total energy describing the static behavior and an Onsager variational principle for the dissipation. These equations can be used to make contact with the approach of macroscopic dynamics used for MRFs throughout the present paper. In particular, the dynamic Eqs. (70) and (71) show a close structural analogy to our Eqs. (B16) and (B17) of Appendix B. In addition, the analog of their dissipative source term for the relative velocity can be found here in Eq. (30). As a result one obtains in the dynamic equations for both velocities a viscous source term containing the relative velocity.

By construction the approach pursued in Refs. [50-52] cannot yield reversible currents carrying a phenomenological parameter. This implies immediately that all effects given in Eqs. (33)-(39) in Sec. II above are absent. In addition, we also note that - not having a magnetization as a dynamic variable, since ERFs were considered-all effects discussed in Sec. II related to the magnetization do not arise. This includes static terms in the magnetization given and analyzed in Eqs. (5)-(12), dissipative terms presented and discussed in Eqs. (21)-(32), as well as all dynamic reversible contributions and their consequences investigated in Eqs. (33)-(39).

\section{SUMMARY AND PERSPECTIVE}

In the present paper a macroscopic model to investigate two-fluid effects for MRFs has been proposed. In order to incorporate the effects of the chains of magnetizable particles, which are observed in experiments, and to capture the solidlike properties, we included as macroscopic variables the magnetization, the strain field, the velocity difference, and the concentration.

We outlined several experimental configurations to test and validate our model. Among them are a relative velocity induced by a magnetic gradient field, the possibility of transverse spatial modulations, and the impossibility of linear velocity profiles in stationary shear flow.

One should mention that while there are no discontinuities in physical properties as the field is increased, there are discontinuous transitions when a MRF is exposed to a shear flow. In these experiments MRF is confined between two parallel plates and a shear flow is imposed perpendicular to 
the imposed magnetic field. If one measures flow curves (i.e., shear stress as a function of shear rate) a jump in the shear stress is observed at a critical shear rate, which corresponds to a transition between a hexagonal array of columns and a lamellar structure, with the layers parallel to the vorticity direction [53-55].

As a next step clearly the macroscopic description of magnetorheological elastomers comes to mind. In this case one has a permanent network from the chemical cross linking in addition to the transient network studied here. In that case relative rotations between the permanent network and the magnetization come into play, as well as relative translations of the two networks.

Since the synthesis of magnetically sensitive liquid crystal materials is currently a hot topic [56-58] we briefly comment on the situation when the isotropic carrier fluid is replaced by a nematic liquid crystal. First of all such a replacement would require the inclusion of the director as an additional variable of the model. This has several consequences on the static as well as dynamic behavior [58-60], particularly regarding the relative orientation of the director and the magnetization. In the dynamics, various new dissipative as well as reversible couplings would appear between the director and the rest of the variables such as magnetization, relative velocity, strain field, and concentration. Finally, nematic liquid crystals are sensitive to electric fields, which means the MRF system can be controlled by both external fields simultaneously.

Finally, another possible direction is the study of two-fluid effects in the area of ferrofluids and ferromagnetic nematics. Here, one has to model the (possibly) discontinuous change of the static and dynamic properties, when the external field is increased.

\section{ACKNOWLEDGMENTS}

Partial support of this work through the Schwerpunktprogramm SPP 1681, "Feldgesteuerte Partikel-MatrixWechselwirkungen: Erzeugung, skalenübergreifende Modellierung und Anwendung magnetischer Hybridmaterialien," of the Deutsche Forschungsgemeinschaft, is gratefully acknowledged, as well as the support of the Slovenian Research Agency, Grants No. P1-0055 and No. J1-9149 (D.S.).

\section{APPENDIX A: THERMODYNAMICS}

We assume the energy to be a first order Eulerian form of the extensive variables and write $[17,20]$

$$
\begin{aligned}
E & =E\left(\rho_{1} V, \rho_{2} V, V, \boldsymbol{g}_{1} V, \boldsymbol{g}_{2} V, \sigma V, \rho_{2} V U_{i j}, \rho_{2} V \boldsymbol{M}, V \boldsymbol{B}\right) \\
& =\varepsilon V=\int \varepsilon d V
\end{aligned}
$$

where the elastic and magnetic degrees of freedom are related to species 2 , only.

For $d V=0$ we get the pressure, $p \equiv-\partial E / \partial V$,

$$
p=-\varepsilon+\mu_{1} \rho_{1}+\bar{\mu}_{2} \rho_{2}+\boldsymbol{v}_{1} \cdot \boldsymbol{g}_{1}+\boldsymbol{v}_{2} \cdot \boldsymbol{g}_{2}+T \sigma+\boldsymbol{H} \cdot \boldsymbol{B},
$$

and the Gibbs relation

$$
\begin{aligned}
d \varepsilon= & \mu_{1} d \rho_{1}+\bar{\mu}_{2} d \rho_{2}+\boldsymbol{v}_{1} \cdot d \boldsymbol{g}_{1}+\boldsymbol{v}_{2} \cdot d \boldsymbol{g}_{2}+T d \sigma \\
& +\boldsymbol{h}^{M} \cdot d \boldsymbol{M}+\boldsymbol{H} \cdot d \boldsymbol{B}+\Phi_{i j} d U_{i j}
\end{aligned}
$$

with the chemical potentials $\mu_{1}, \bar{\mu}_{2}=\mu_{2}+\rho_{2}^{-1} h_{i}^{M} M_{i}+$ $\rho_{2}^{-1} \Phi_{i j} U_{i j}$, the velocities $v_{i}^{(1)}$ and $v_{2}^{(2)}$, and $T, h_{i}^{M}, H_{i}$, and $\Phi_{i j}$ as the appropriate conjugate quantities.

Expressions (A3) and (A4) can easily be rewritten in terms of the variables $\left\{g_{i}, w_{i}, \rho, \phi\right\}$, used in the main text, and are shown as Eqs. (1) and (2). The conjugate quantities are related by

$$
\begin{gathered}
\mu=\phi \mu_{1}+(1-\phi) \bar{\mu}_{2}+\rho^{-1} h_{i}^{w} w_{i}, \\
\Pi=\rho\left(\mu_{1}-\bar{\mu}_{2}\right)+\rho v_{i} w_{i}+\left(\rho_{2}-\rho_{1}\right) \boldsymbol{w}^{2}, \\
v_{i}=\rho^{-1} g_{i}, \\
h_{i}^{w}=\rho \phi(1-\phi) w_{i} .
\end{gathered}
$$

In the main text we do not need $\mu$ and we neglect the quadratic velocity terms in $\Pi$, which can then be interpreted as the osmotic pressure, related to the difference of the chemical potentials of the two species. Equations (A6) and (A7) are the result of the assumption that $\boldsymbol{v}_{1,2}$ are the conjugates to $\boldsymbol{g}_{1,2}$. They coincide with the results of Eq. (4), which are derived from the kinetic energy, Eq. (3).

\section{APPENDIX B: GENERAL TRANSPORT DERIVATIVES}

In a two-fluid hydrodynamic description the velocity with which a variable is transported is not fixed by first principles, but depends on the material properties [17]. These transport velocities can be different for different variables, and even for a given variable, e.g. the strain tensor, the advective velocity can be different from the convective one. The only restriction is reversibility, i.e., the transport contributions are reversible and must not contribute to the dissipation function. We first set up the most general expressions for the transport terms allowed by thermodynamics, and then choose special values for the material dependent parts, such that variables belonging to subsystem 1 or 2 are transported by $\boldsymbol{v}_{1}$ or $\boldsymbol{v}_{2}$, respectively. This results in a considerable simplification and leads to the approximation used in the main body of the paper. By this procedure it is made sure that the thermodynamics is still obeyed.

We start with the set of dynamic equations for all variables involved and take the mean velocity as the transport velocity for all of them and get $[17,19-21,25,26]$

$$
\begin{gathered}
\dot{\rho}+\nabla_{j}\left(\rho v_{j}\right)=0, \\
\dot{\phi}+v_{j} \nabla_{j} \phi+\rho^{-1} \nabla_{i} j_{i}^{\phi}=0, \\
\dot{\varepsilon}+\nabla_{j}(\varepsilon+p) v_{j}+\nabla_{i} j_{i}^{\varepsilon}=0, \\
\dot{\sigma}+\nabla_{j}\left(\sigma v_{j}+j_{i}^{\sigma}\right)=2 R / T, \\
\dot{w}_{i}+v_{j} \nabla_{j} w_{i}+\nabla_{i} \Pi+Y_{i}=0,
\end{gathered}
$$




$$
\begin{gathered}
\dot{g}_{i}+\nabla_{j} g_{i} v_{j}+\nabla_{j}\left(-\Phi_{i j}+2 \Phi_{j k} U_{i k}\right)+\nabla_{i} p \\
-\frac{1}{2} \nabla_{j}\left(B_{i} H_{j}+B_{j} H_{i}\right)+\nabla_{j} \sigma_{i j}=0, \\
\dot{M}_{i}+v_{j} \nabla_{j} M_{i}+\epsilon_{i j k} M_{j} \omega_{k}+X_{i}=0, \\
\dot{U}_{i j}+v_{k} \nabla_{k} U_{i j}+U_{k j} \nabla_{i} v_{k}+U_{k i} \nabla_{j} v_{k}-A_{i j}+Z_{i j}=0,
\end{gathered}
$$

where $A_{i j}=\left(\nabla_{i} v_{j}+\nabla_{j} v_{i}\right) / 2$ is the symmetric gradient of the mean velocity and the vorticity $2 \omega_{i}=\epsilon_{i j k} \nabla_{j} v_{k}$ corresponds to its antisymmetric gradient. In this description also $\rho_{1,2}$ and $v_{i}^{(1,2)}$ are convected by the mean velocity. The material dependent contributions are described by the phenomenological currents $j_{i}^{\phi}, j_{i}^{\varepsilon}, j_{i}^{\sigma}, Y_{i}, \sigma_{i j}, X_{i}$, and $Z_{i j}$.

The nonphenomenological contributions, shown explicitly in Eqs. (B1)-(B8), all together give zero entropy production $R=0$, when put into the Gibbs relation Eq. (1). As a result there are some contributions not directly related to transport, e.g., $\nabla_{j}\left(p v_{j}\right)$ in Eq. (B3), $\nabla_{j} \Pi$ in Eq. (B5), $\nabla_{i} p$, and the linear and nonlinear elastic stresses and the Maxwell stress in Eq. (B6). The special form of Eq. (B2) is due to the individual conservation of the two densities $\rho_{1,2}$.

In a one-fluid system the transport terms would be fixed, since the phenomenological currents must not contain the velocity due to Galilean invariance. In a two-fluid system, however, a dependence on the relative velocity $w_{i}$ is possible, which can change the effective transport velocity. For the relative velocity dependent parts of the reversible currents we find using time-reversal and spatial rotational symmetry [17]

$$
\begin{gathered}
Z_{i j}^{(\operatorname{rev} T)}=\beta_{1}\left(\nabla_{i} h_{j}^{w}+\nabla_{j} h_{i}^{w}\right)+\beta_{6} h_{k}^{w} \nabla_{k} U_{i j} \\
+\beta_{7}\left(U_{k j} \nabla_{i} h_{k}^{w}+U_{k i} \nabla_{j} h_{k}^{w}\right), \\
\sigma_{i j}^{(\operatorname{rev} T)}=2 \beta_{2} h_{i}^{w} w_{j}, \\
Y_{i}^{(\operatorname{rev} T)}=2 \nabla_{j}\left(\beta_{1} \Phi_{i j}\right)+\beta_{2} w_{j}\left(\nabla_{j} v_{i}+\nabla_{i} v_{j}\right) \\
+\beta_{3} h_{j}^{w}\left(\nabla_{j} w_{i}-\nabla_{i} w_{j}\right)+\beta_{4} w_{j}\left(\nabla_{j} v_{i}-\nabla_{i} v_{j}\right) \\
+\beta_{5} \nabla_{i} \Pi-\beta_{6} \Phi_{k j} \nabla_{i} U_{k j} \\
+\nabla_{j} \beta_{7}\left(\Phi_{k j} U_{i k}+\Phi_{k i} U_{j k}\right)-\beta_{8} h_{j}^{M} \nabla_{i} M_{j} \\
+\beta_{9} \nabla_{k}\left(h_{i}^{M} M_{k}-h_{k}^{M} M_{i}\right), \\
X_{i}^{(\operatorname{rev} T)}=\beta_{8} h_{j}^{w} \nabla_{j} M_{i}+M_{k}\left(\nabla_{k} \beta_{9} h_{i}^{w}-\nabla_{i} \beta_{9} h_{k}^{w}\right), \\
j_{i}^{(\phi, r e v T)}=\beta_{5} h_{i}^{w},
\end{gathered}
$$

where we have omitted the thermal degree of freedom, since we assume that it is convected by the mean velocity anyhow.

We will now specialize our system such that the variables $\left\{\rho_{1}, v_{i}^{(1)}\right\}$ and $\left\{\rho_{2}, v_{i}^{(2)}, M_{i}, U_{i j}\right\}$ are transported by $v_{i}^{(1)}$ and $v_{i}^{(2)}$, respectively, by assigning appropriate values to the various reversible transport parameters $\beta_{n}$. First, we take $\beta_{5}=1$, which leads to the desired result [17]:

$$
\begin{aligned}
& \dot{\rho}_{1}+\nabla_{i} \rho_{1} v_{i}^{(1)}+\nabla_{i} j_{i}^{\phi}=0, \\
& \dot{\rho}_{2}+\nabla_{i} \rho_{2} v_{i}^{(2)}-\nabla_{i} j_{i}^{\phi}=0 .
\end{aligned}
$$

With $\beta_{5}=1$, Eq. (B2) gives the final result, Eq. (14) in Sec. II B. In order to replace in the transport dynamics of the strain tensor, Eq. (B8), the mean velocity by $v_{i}^{(2)}$, we take $2 \beta_{1}=-\beta_{6}=-\beta_{7}=1 / \rho_{2}$ [17]. Similarly, for the magnetization we choose $\beta_{9}=-\beta_{8}=1 / \rho_{2}$. The resulting expressions are shown as Eqs. (20) and (19) in Sec. II B.

Somewhat more difficult is the assignment for $\beta_{2,3,4}$. As is discussed in Ref. [17] the choice $\beta_{3}=\left(1 / \rho_{1}\right)-\left(1 / \rho_{2}\right)$ leads to

$$
\begin{aligned}
& \dot{g}_{i}^{(1)}+\nabla_{j} g_{i}^{(1)} v_{j}^{(1)}+\frac{\rho_{1}}{\rho} \nabla_{j} \sigma_{i j}+X_{i}^{(1)}=0, \\
& \dot{g}_{i}^{(2)}+\nabla_{j} g_{i}^{(2)} v_{j}^{(2)}+\frac{\rho_{2}}{\rho} \nabla_{j} \sigma_{i j}+X_{i}^{(2)}=0,
\end{aligned}
$$

and the value $\beta_{2}=1 / 2$ results in a contribution $h_{i}^{w} w_{j}$ in Eq. (B11) that finally gives the correct momentum current density due to flow in Eq. (18) in Sec. II B. For $\beta_{4}$ we take $\beta_{4}=1 / 2$ to ensure that there are no additional convection terms hidden in $X_{i}^{(1,2)}$. The various contributions in Eq. (B12) lead to a large number of additional terms to be found in the dynamics of the relative velocity Eq. (17).

\section{APPENDIX C: VORTICITY OF $M$ AND THE RELATIVE VELOCITY}

In writing down Eq. (5) of the main text a coupling between the relative velocity and magnetization gradients, $\sim \epsilon_{i j k} w_{i} \nabla_{j} M_{k}$, allowed by symmetry, has been discarded, since it is incompatible with the assumption that $g_{1}$ and $g_{2}$ are only related to $\boldsymbol{v}_{1}$ and $\boldsymbol{v}_{2}$, respectively, in our simplified model. Here we analyze some implications of such a term and its structural similarity to terms associated with the $\hat{l}$ vector in superfluid ${ }^{3} \mathrm{He}$.

In the energy Eq. (5) this contribution takes the uniaxial form $\varepsilon_{2}^{\text {vort }}=f_{i j k} w_{i} \nabla_{j} M_{k}$ with $f_{i j k}=\left(f_{1} M_{i} M_{l}+f_{2} \delta_{i l}\right) \epsilon_{l j k}$ and the thermodynamic forces of the magnetization and the relative velocity get the additional linear contributions

$$
\begin{gathered}
h_{i}^{M}=\frac{\delta \varepsilon_{2}^{\text {vort }}}{\delta M_{i}}=-\nabla_{j}\left(f_{i j k} w_{k}\right), \\
h_{i}^{w}=\frac{\partial \varepsilon_{2}^{\text {vort }}}{\partial w_{i}}=f_{i j k} \nabla_{j} M_{k} .
\end{gathered}
$$

These two additions lead, together with Eqs. (4) and (10) in the case of no field, no strain, and no concentration, to the linearized equilibrium conditions $-f_{2} \operatorname{curl} \boldsymbol{w}+\alpha \boldsymbol{M}=0$ and $f_{2} \operatorname{curl} \boldsymbol{M}+\rho \phi(1-\phi) \boldsymbol{w}=0$, which allow for a spatial inhomogeneous helical structure with $\boldsymbol{w} \sim(-\cos k z, \sin k z, 0) \sim$ $\boldsymbol{M}$. Being a linear gradient term, $\varepsilon_{2}^{\text {vort }}$ can be negative for this helical state and, therefore, be lower than the energy of the homogeneous state. However, since helical flow of $w_{i}$ is involved, this state is not a true static one, but involves dissipation as well and the helical state is not stable. But as a transient it might be possible.

Another possibility for experiments would be to induce a magnetic vortex $M_{0} \hat{\boldsymbol{e}}_{\varphi}(r, \varphi$, and $z$ are cylindrical coordinates) by an external field via Eq. (10). By Eq. (4) then a flow $\boldsymbol{w} \sim \hat{\boldsymbol{e}}_{z} / r$ follows. It seems to be difficult to produce such an external vortex magnetic field. Vice versa, an externally induced vortex of $w_{i}$ would induce a finite magnetizationbut there is no external field that can induce a $w_{i}$ flow. 
This kind of coupling is rather similar to one of the couplings appearing in superfluid ${ }^{3} \mathrm{He}-\mathrm{A}$ first introduced into macroscopic dynamics in Ref. [15]. In this system one defines an axial vector I parallel to the direction of the net orbital momentum of the helium pairs. This vector does not commute with the total angular momentum vector and therefore this variable breaks the continuous rotational symmetry spontaneously similar to the magnetization in our system. The source free part of the momentum density of ${ }^{3} \mathrm{He}-\mathrm{A}$ is then proportional to the curl of this vector $\mathbf{l}$. For ${ }^{3} \mathrm{He}$ this coupling has also significant consequence for the static correlation functions involving different orientations of the $\mathbf{l}$ vector [16].

\section{APPENDIX D: FIELD DEPENDENT GROUND STATE}

In the absence of a magnetic field the equilibrium magnetization is zero, $M_{i}=0$, and there is no strain, $U_{i j}=0$, and no concentration variation $\bar{\phi} \equiv \phi-\rho_{2} / \rho=0$. For a finite external field the new ground state is given by a finite magnetization due to the direct coupling $\sim H_{i} M_{i}$, by a nonzero strain $U_{k k}$ due to magnetostriction and by a finite $\bar{\phi}$ due to the $\chi_{m}$ coupling. These ground-state values are found by the condition that the first variations of $\varepsilon_{2}$, Eq. (5), with respect to all variables have to vanish and we get the implicit expressions

$$
\begin{gathered}
\mu_{0} H_{i}=\left(\tilde{\alpha}+\tilde{\beta} M_{0}^{2}\right) M_{i}^{0}, \\
\chi_{\phi} \bar{\phi}_{0}=-\left(\chi_{u} U_{k k}^{0}+\chi_{m}\right) M_{0}^{2}, \\
2 U_{k k}^{0}=\frac{3 \gamma_{1}+2 \gamma_{2}}{3 c_{1}+2 c_{2}}+O\left(M_{0}^{2}\right), \\
4 U_{z z}^{0}=\left(\gamma_{1}+2 \gamma_{2}-2 c_{1} U_{k k}^{0}\right) / c_{2}, \\
2 U_{i j}^{0}=\delta_{i j}\left(U_{k k}^{0}-U_{z z}^{0}\right)-\delta_{i z} \delta_{j z}\left(U_{k k}^{0}-3 U_{z z}^{0}\right), \\
2 U_{i j}^{0} U_{i j}^{0}=\left(U_{k k}^{0}-U_{z z}^{0}\right)^{2}+2 U_{z z}^{0} U_{z z}^{0},
\end{gathered}
$$

where the field $H_{i}=H \delta_{i z}$ defines the $z$ direction and with $\tilde{\alpha}=$ $\alpha+c_{1} U_{k k}^{0} U_{j j}^{0}+2 c_{2} U_{i j}^{0} U_{i j}^{0}-\left(\gamma_{1} / 2\right) U_{k k}^{0}-\gamma_{2} U_{z z}^{0}$ and $\tilde{\beta}=\beta-$ $\left(2 / \chi_{\phi}\right)\left(\chi_{u} U_{k k}^{0}+\chi_{m}\right)^{2}$.

The ground-state strains $U_{k k}^{0}$ and $U_{z z}^{0}$ do not vanish in the zero field limit. On the other hand, starting with zero field, there are no strains (since the elastic moduli are $\sim M^{2}$ ) and no magnetostriction is present. Therefore, the equations as presented here are not suitable for the case of very low external fields. The problem comes from our choice of $\tilde{c}_{1,2} \sim M_{0}^{2}$, which leads to field independent magnetostrictive deformation, since magnetostriction is also $\sim M_{0}^{2}$.

The remedy of this problem is the reasonable assumption that very small fields, $H<H^{*}$, cannot produce an elastic structure. Therefore, instead of Eq. (8) we can write

$$
\tilde{c}_{1,2}=\left\{\begin{array}{ll}
0 & \text { for } 0<H<H^{*} \\
M^{2} c_{1,2} & \text { for } H>H^{*}
\end{array},\right.
$$

which leads to $U_{i j}^{0}=0$ for $H \rightarrow 0$. For $H>H^{*}$ the description used in the main text remains unchanged.

Rewriting Eq. (5) in terms of the deviation from the new ground state, $\delta M_{i} \equiv M_{i}-M_{i}^{0}, \delta U_{i j} \equiv U_{i j}-U_{i j}^{0}$, and $\delta \phi \equiv$ $\bar{\phi}-\bar{\phi}_{0}$, we get for the relevant conjugates in linear order (and for isotropic elasticity)

$$
\begin{aligned}
h_{i}^{M}=\frac{\delta \varepsilon}{\delta M_{i}}= & \alpha_{\mathrm{eff}} \delta M_{i}-2 \gamma_{2} U_{i j}^{0} \delta M_{j}+4 c_{2} M_{i}^{0} U_{k l}^{0} \delta U_{k l} \\
& -\gamma_{1}^{(\mathrm{eff})} M_{i}^{0} \delta U_{k k}-2 \gamma_{2} M_{j}^{0} \delta U_{i j} \\
& +2\left(\chi_{m}+\chi_{u} U_{k k}^{0}\right) M_{i}^{0} \delta \phi, \\
\Phi_{i j}=\frac{\delta \varepsilon}{\delta U_{i j}}= & c_{1} M_{0}^{2} \delta_{i j} \delta U_{k k}+2 c_{2} M_{0}^{2} \delta U_{i j}+\chi_{u} M_{0}^{2} \delta_{i j} \delta \phi \\
& -\gamma_{1}^{(\mathrm{eff})} \delta_{i j} M_{p}^{0} \delta M_{p}-\gamma_{2}\left(M_{i}^{0} \delta M_{j}+M_{j}^{0} \delta M_{i}\right) \\
& +4 c_{2} U_{i j}^{0} M_{k}^{0} \delta M_{k}, \\
\delta \Pi=\frac{\delta \varepsilon}{\delta \phi}=\chi_{\phi} \delta \phi & +\chi_{u} M_{0}^{2} \delta U_{k k}+2\left(\chi_{m}+\chi_{u} U_{k k}^{0}\right) M_{i}^{0} \delta M_{i},
\end{aligned}
$$

where $\delta \Pi=\Pi-\rho\left(\mu_{1}-\bar{\mu}_{2}\right)$ is the excess osmotic pressure [see Eq. (A7)]. The coefficients $\gamma_{1}^{(\text {eff })}=\gamma_{1}-2 c_{1} U_{k k}^{0}-2 \chi_{u} \bar{\phi}_{0}$ and $\quad \alpha_{\text {eff }}=\alpha+3 \beta M_{0}^{2}+c_{i j k l} U_{i j}^{0} U_{k l}^{0}-\gamma_{1} U_{k k}^{0}+2 \chi_{u} \bar{\phi}_{0} U_{k k}^{0}+$ $2 \chi_{m} \bar{\phi}_{0}$ are complicated functions of many static susceptibilities, with their functional dependence on the external field strength generally taken from experiments. In the ground state the variables $\delta M_{i}, \delta U_{i j}$, and $\delta \phi$ vanish by definition and therefore the conjugates $h_{i}^{M}, \delta \Pi$, and $\Phi_{i j}$ also vanish.

\section{APPENDIX E: REDUCTION TO THE ONE-FLUID CASE}

The equations derived above are the most general twofluid equations for MRF (assuming the total mass density to be constant and neglecting the thermal degree of freedom). Of course, these equations must contain the one-fluid description [18] as a special case. Setting $w_{i}=0$, meaning $v_{i}=v_{i}^{(1)}=v_{i}^{(2)}$, the conjugate $h_{i}^{w} \equiv 0$ fulfils the static Eq. (4) automatically. However, we have to require that $w_{i}=0$ holds for all times, i.e. $\dot{w}_{i}=0$. In the long wavelength limit (all gradients are zero) the relative velocity obeys the linearized relaxation equation [see Eqs. (17) and (30)]

$$
\dot{w}_{i}+\frac{\rho_{1} \rho_{2}}{\rho} \xi_{i j} w_{j}=0
$$

If the relaxation rates, $\xi$ and $\xi_{\perp}$, are much shorter than the relevant time scales of the other macroscopic variables, $w_{i}=0$ and can be dropped in all other currents.

In the general case, $\dot{w}_{i}=0$ requires, including Eq. (34),

$$
w_{i} \sim\left(-\nabla_{i} \Pi-\frac{1}{\rho_{2}} \nabla_{j} \Phi_{i j}+\gamma_{i j k} \nabla_{j} h_{k}^{M}+v_{i j k l}^{(c)} \nabla_{j} A_{k l}\right) \text {. }
$$

Replacing $w_{i}$ in the dynamic equations for the other variables-Eqs. (19), (31), and (35) for $M_{i}$; Eqs. (18), (28), and (36) for $g_{i}$; and Eqs. (20), (29), and (37) for $U_{i j}$-leads to contributions, which are two orders higher in the gradients than those already present (and can therefore be neglected). The only exception is the term $\alpha_{i j k}$ in Eq. (31), that gets replaced by $\alpha_{i j k}+\gamma_{p j i} \xi_{k p}^{-1}$ with $\xi_{k p}^{-1} \xi_{k j}=\delta_{p j}$. These are then the equations for a binary MRF that is described by one velocity but two densities. 
The final step to the one-fluid MRF is the elimination of one density. With, e.g., $\rho_{1}=\rho_{2}$ there is $\bar{\phi}=1 / 2$ and $\delta \phi=0$. This eliminates the concentration degree of freedom from the statics, Eq. (5). Since also $\dot{\phi}=0$ is required, the concentration current, the sum of Eqs. (32) and (33), has to be constant. This leads to a replacement of $\nabla_{j} \Pi=-D_{i j}^{-1} \alpha_{k i l} \nabla_{l} h_{k}^{M}$ (with $D_{i k}^{-1} D_{i j}=\delta_{k j}$ ) in Eq. (31), where it can then be neglected when compared with $b_{i j}^{D} h_{j}^{M}$. Note that also the equilibrium value of $\Pi=\rho\left(\mu_{1}-\bar{\mu}_{2}\right)$ vanishes.
[1] D. A. Drew and S. L. Passman, Theory of Multi-Component Fluids, Applied Mathematical Sciences Vol. 135 (Springer, New York, 1999).

[2] P. Hebraud, F. Lequeux, and J. F. Palierne, Langmuir 16, 8296 (2000).

[3] I. A. Kadoma and J. W. van Egmond, Phys. Rev. Lett. 80, 5679 (1998).

[4] D. Lhuillier, J. Non-Newtonian Fluid Mech. 96, 19 (2001).

[5] A. Onuki, Phys. Rev. Lett. 62, 2472 (1989).

[6] S. T. Milner, Phys. Rev. Lett. 66, 1477 (1991).

[7] E. Helfand and G. H. Fredrickson, Phys. Rev. Lett. 62, 2468 (1989).

[8] S. Saito, M. Takenaka, N. Toyoda, and T. Hashimoto, Macromol. 34, 6461 (2001).

[9] M. Doi and A. Onuki, J. Phys. II (France) 2, 1631 (1992).

[10] A. Onuki, R. Yamamoto, and T. Taniguchi, J. Phys. II (France) 7, 295 (1997).

[11] T. Sun, A. C. Balazs, and D. Jasnow, Phys. Rev. E 59, 603 (1999).

[12] T. Araki and T. Tanaka, Macromol. 34, 1953 (2001).

[13] H. Pleiner, D. Svenšek, and H. R. Brand, Eur. Phys. J. E 36, 135 (2013).

[14] I. M. Khalatnikov, Introduction to the Theory of Superfluidity (Benjamin, New York, 1965).

[15] R. Graham, Phys. Rev. Lett. 33, 1431 (1974).

[16] H. R. Brand, M. Dörfle, and R. Graham, Ann. Phys. (NY) 119, 434 (1979).

[17] H. Pleiner and J. L. Harden, in Nonlinear problems of continuum mechanics, special issue of notices of universities, South of Russia, Natural Sci. 46 (2003); arXiv:cond-mat/0404134.

[18] T. Potisk, D. Svenšek, H. Pleiner and H. R. Brand, J. Chem. Phys. 150, 174901 (2019).

[19] H. Pleiner and J. L. Harden, AIP Proc. 708, 46 (2004).

[20] H. Pleiner and H. R. Brand, in Pattern Formation in Liquid Crystals, edited by A. Buka and L. Kramer (Springer, New York, 1996).

[21] E. Jarkova, H. Pleiner, H.-W. Müller, and H. R. Brand, Phys. Rev. E 68, 041706 (2003).

[22] S. Bohlius, H. R. Brand, and H. Pleiner, Phys. Rev. E 70, 061411 (2004).

[23] D. Forster, Hydrodynamic Fluctuations, Broken Symmetry and Correlation Functions (Benjamin, New York, 1975).

[24] P. C. Martin, O. Parodi, and P. Pershan, Phys. Rev. A 6, 2401 (1972).

[25] H. Temmen, H. Pleiner, M. Liu, and H. R. Brand, Phys. Rev. Lett. 84, 3228 (2000).

[26] H. Pleiner, M. Liu, and H. R. Brand, Rheol. Acta 39, 560 (2000).

[27] M. Grmela, Phys. Lett. A 296, 97 (2002).
[28] H. R. Brand, H. Pleiner, and D. Svenšek, Rheol. Acta 57, 773 (2018).

[29] S. R. de Groot and P. Mazur, Nonequilibrium Thermodynamics (North-Holland, Amsterdam, 1962).

[30] W. H. Li, H. Du, G. Chen, and S. H. Yeo, Mater. Sci. Eng. A 333, 368 (2002).

[31] W. H. Li, H. Du, G. Chen, S. H. Yeo, and N. Q. Guo, Smart Mater. Struct. 11, 209 (2002).

[32] J. Claracq, J. Sarrazin, and J.-P. Montfort, Rheol. Acta 43, 38 (2004).

[33] Y. Otsubo and K. Edamura, J. Rheol. 38, 1721 (1994).

[34] Z. Wang, K. Shahrivar, and J. de Vicente, J. Rheol. 58, 1725 (2014).

[35] K. D. Weiss, J. D. Carlson, and D. A. Nixon, J. Intell. Mater. Syst. Struct. 5, 772 (1994).

[36] B. D. Chin, J. H. Park, M. H. Kwon, and O. O. Park, Rheol. Acta 40, 211 (2001).

[37] H. R. Brand, H. Pleiner, and D. Svenšek, Eur. Phys. J. E 34, 128 (2011).

[38] H. W. Müller and M. Liu, Phys. Rev. E 64, 061405 (2001).

[39] G. Bossis, O. Volkova, S. Lacis, and A. Meunier, Lect. Notes Phys. 594, 202 (2002).

[40] S. Odenbach, Magnetoviscous Effects in Ferrofluids, Lecture Notes in Physics Monographs (Springer-Verlag, Berlin, 2002).

[41] W. H. Li, P. Q. Zhang, X. L. Gong, and P. B. Kosasih, Int. J. Modern Phys. B 19, 1198 (2005).

[42] P. Kuzhir, M. T. López-López, and G. Bossis, Phys. Fluids 21, 053101 (2009)

[43] B. Horvath and I. Szalai, Phys. Rev. E 86, 061403 (2012).

[44] T. Potisk, D. Svenšek, H. Pleiner, and H. R. Brand (unpublished).

[45] K. von Pfeil, M. D. Graham, D. J. Klingenberg, and J. F. Morris, Phys. Rev. Lett. 88, 188301 (2002).

[46] K. von Pfeil, M. D. Graham, D. J. Klingenberg, and J. F. Morris, J. Appl. Phys. 93, 5769 (2003).

[47] S. Zhu, Q. Luo, J. Liu, and T. Fujita, Adv. Mat. Sci. Eng. Hindawi 2016, 5108401 (2016).

[48] J. Zheng, W.-J. Fu, and L.-W. Zhou, Chin. Phys. Lett. 30, 094701 (2013).

[49] C. Li, J. Huang, Q. Tang, J. Huang, J. Zhang, and L. Zhou, Soft Matter 8, 5250 (2012).

[50] J. W. Zhang, X. Q. Gong, C. Liu, W. Wen, and P. Sheng, Phys. Rev. Lett. 101, 194503 (2008).

[51] P. Sheng and W. Wen, Solid State Commun. 150, 1023 (2010).

[52] P. Sheng and W. Wen, Annu. Rev. Fluid Mech. 44, 143 (2012) including Supplemental Material.

[53] S. Cutillas and G. Bossis, Europhys. Lett. 40, 465 (1997). 
[54] S. Cutillas, G. Bossis, and A. Cebers, Phys. Rev. E 57, 804 (1998).

[55] O. Volkova, S. Cutillas, and G. Bossis, Phys. Rev. Lett. 82, 233 (1999).

[56] A. Mertelj, D. Lisjak, M. Drofenik, and M. Čopič, Nature (London) 504, 237 (2013).

[57] A. Mertelj, N. Osterman, D. Lisjak, and M. Čopič, Soft Matter 10, 9065 (2014).
[58] T. Potisk, D. Svenšek, H. R. Brand, H. Pleiner, D. Lisjak, N. Osterman, and A. Mertelj, Phys. Rev. Lett. 119, 097802 (2017).

[59] T. Potisk, A. Mertelj, N. Sebastian, N. Osterman, D. Lisjak, H. R. Brand, H. Pleiner, and D. Svenšek, Phys. Rev. E 97, 012701 (2018).

[60] T. Potisk, H. Pleiner, D. Svenšek, and H. R. Brand, Phys. Rev. E 97, 042705 (2018). 